\title{
Spin Motion through Helical Dipole Magnets
}

\author{
M. J. Syphers
}

February 12, 1996

The equation of motion of the spin vector $\vec{S}$ through a magnetic field is given by

$$
\frac{d \vec{S}}{d s}=\vec{S} \times \vec{\Omega}
$$

where

$$
\vec{\Omega} \equiv \frac{1}{B \rho}\left[(1+G \gamma) \overrightarrow{B_{\perp}}+(1+G) \overrightarrow{B_{\|}}\right]
$$

While this equation can be integrated numerically through a helical dipole field ${ }^{1}$, it is sometimes more convenient for spin tracking and for systems design to model the spin behavior using $3 \times 3$ matrices operating on the spin vector $\vec{S}$. In the code $\mathrm{SPINK}^{2}$, a Snake is modeled as a single rotation of $180^{\circ}$ about a horizontal axis. To perform sensitivity analyses on the RHIC Snake and Rotator systems, one would like to model individually each helical dipole magnet of a Snake or Rotator.

To solve for the spin motion through a helical dipole magnet, it is convenient to switch to the spinor representation. (I'll follow the formuation used by Courant ${ }^{3}$.) Any spin vector $\vec{S}$ can be represented by

$$
\vec{S}=\psi^{\dagger} \vec{\sigma} \psi
$$

where $\vec{\sigma}$ is made up of the Pauli spin matrices:

$$
\begin{aligned}
\vec{\sigma} & =\sigma_{1} \hat{x}_{1}+\sigma_{2} \hat{x}_{2}+\sigma_{3} \hat{x}_{3} \\
& =\left(\begin{array}{ll}
0 & 1 \\
1 & 0
\end{array}\right) \hat{x}_{1}+\left(\begin{array}{cc}
0 & -i \\
i & 0
\end{array}\right) \hat{x}_{2}+\left(\begin{array}{cc}
1 & 0 \\
0 & -1
\end{array}\right) \hat{x}_{3}
\end{aligned}
$$

and $\psi$ is a 2-component vector, or "spinor." Plugging this definition into the the equation of motion of the spin vector, we find that

$$
\frac{d \psi}{d s}=\frac{i}{2}(\vec{\sigma} \cdot \vec{\Omega}) \psi
$$

\footnotetext{
${ }^{1}$ A. Luccio, "Numerical Studies of Siberian Snakes and Spin Rotators for RHIC," BNL-52461 $(\mathrm{SN} / 8)$.

${ }^{2}$ A. Luccio, "Numerical Spin Tracking in a Synchrotron," BNL-52481 (SN/11).

${ }^{3}$ E. Courant, "Hybrid Helical Snakes and Rotators for RHIC," BNL-61920 (SN/10).
} 
For the case where $\vec{\Omega}=$ constant, the solution for $\psi$ is just

$$
\psi(s)=\exp [(i / 2)(\vec{\sigma} \cdot \vec{\Omega}) s] \times(\text { constant })
$$

or in matrix form:

$$
\psi\left(s_{2}\right)=e^{\frac{i}{2}(\vec{\sigma} \cdot \vec{\Omega})\left(s_{2}-s_{1}\right)} \psi\left(s_{1}\right) .
$$

Any rotation through an angle $\theta$ about an axis given by the unit vector $\hat{n}$ can be represented by ${ }^{4}$

$$
M_{\theta}=e^{-\frac{i}{2} \theta(\vec{\sigma} \cdot \hat{n})}
$$

and so the quantity $(\vec{\sigma} \cdot \vec{\Omega})\left(s_{2}-s_{1}\right)$ above can provide us with the angle and axis of rotation for a helical dipole.

As a simple example, consider a horizontal dipole field over a length $L$, producing a vertical bend in a proton's trajectory. Then $\vec{\Omega}=(1+G \gamma) B_{0} /(B \rho) \hat{x}_{2}$, where we are taking the longitudinal axis as the "1"-direction, the horizontal axis as the "2"direction, and the vertical axis as the " 3 "-direction. Then, $\vec{\sigma} \cdot \vec{\Omega}=\kappa \sigma_{2}$, where $\kappa \equiv(1+G \gamma) B_{0} /(B \rho)$. Looking at this in terms of a matrix of the form $M_{\theta}$ above, this says that the spin vector is rotated through an angle $\theta=(1+G \gamma) B_{0} L /(B \rho)$ about the horizontal ("2") axis as the particle passes through the dipole field, which is the intuitive result from Equation 1.

In a similar manner, the spin motion through a helical dipole magnet was described by Courant $^{5}$ and can be written as:

$$
M_{h r}=e^{\frac{i}{2}\left(k L \sigma_{1}+\kappa L \sigma_{2}\right)}
$$

where the subscript "hr" denotes that this is the matrix for a Rotator-style helical dipole (beginning and ending with the field horizontal). For this complete helix, $k L=2 \pi$ and hence in real space the spin motion through this magnet is equivalent to a rotation through an angle

$$
\mu=-2 \pi \sqrt{1+\left(\frac{\kappa}{k}\right)^{2}}
$$

about an axis

$$
\hat{n}=\frac{\hat{x}_{1}+\left(\frac{\kappa}{k}\right) \hat{x}_{2}}{\sqrt{1+\left(\frac{\kappa}{k}\right)^{2}}} .
$$

The unit vector $\hat{n}$ is in the $x$-z plane, at an angle $\phi=\tan ^{-1}(\kappa / k)$ to the $z$-axis. The rotation of the spin vector $\vec{S}$ is indicated in Figure 1. With this figure in mind, it

\footnotetext{
${ }^{4}$ c.f., E. Merzbacher, "Quantum Mechanics," John Wiley \& Sons, (1970) p. 266.

${ }^{5}$ E. Courant, SN/10.
} 


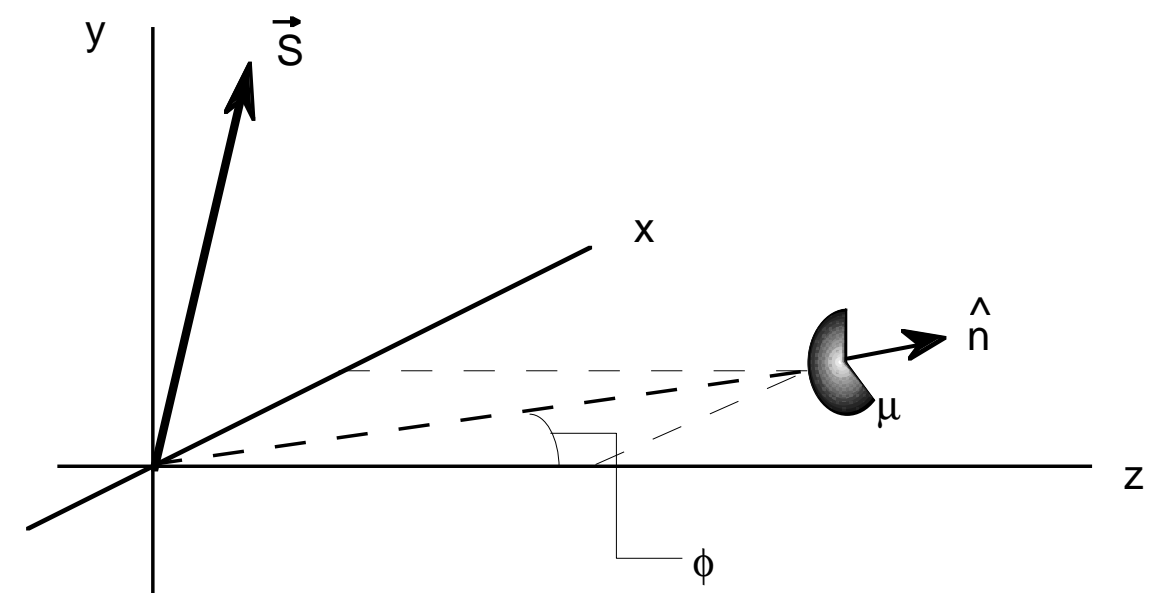

Figure 1: Rotation of spin vector $\vec{S}$ through an angle $\mu$ about an axis $\hat{n}$. For a "Rotator" helical dipole magnet, $\hat{n}$ is in the horizontal plane at an angle $\phi$ with the $z$-axis.

is easy to produce the spacial transformation of the spin vector through a Rotator magnet using a series of rotations:

$$
\begin{aligned}
M_{h r}^{(3)} & =\left(\begin{array}{ccc}
\cos \phi & 0 & \sin \phi \\
0 & 1 & 0 \\
-\sin \phi & 0 & \cos \phi
\end{array}\right)\left(\begin{array}{ccc}
\cos \mu & -\sin \mu & 0 \\
\sin \mu & \cos \mu & 0 \\
0 & 0 & 1
\end{array}\right)\left(\begin{array}{ccc}
\cos \phi & 0 & -\sin \phi \\
0 & 1 & 0 \\
\sin \phi & 0 & \cos \phi
\end{array}\right) \\
& =\left(\begin{array}{ccc}
\cos ^{2} \phi \cos \mu+\sin ^{2} \phi & -\cos \phi \sin \mu & \cos \phi \sin \phi(1-\cos \mu) \\
\cos \phi \sin \mu & \cos \mu & -\sin \phi \sin \mu \\
\cos \phi \sin \phi(1-\cos \mu) & \sin \phi \sin \mu & \sin ^{2} \phi \cos \mu+\cos ^{2} \phi
\end{array}\right)
\end{aligned}
$$

Where

$$
\left(\begin{array}{c}
S_{x} \\
S_{y} \\
S_{z}
\end{array}\right)_{\text {out }}=M_{h r}^{(3)}\left(\begin{array}{c}
S_{x} \\
S_{y} \\
S_{z}
\end{array}\right)_{\text {in }}
$$

through a Rotator helical dipole.

For a Snake-style magnet (beginning and ending with the field vertical), the spinor matrix would be

$$
M_{h s}=e^{\frac{i}{2}\left(k L \sigma_{1}+\kappa L \sigma_{3}\right)}
$$

and thus the rotation would be through an angle

$$
\mu=-2 \pi \sqrt{1+\left(\frac{\kappa}{k}\right)^{2}}
$$




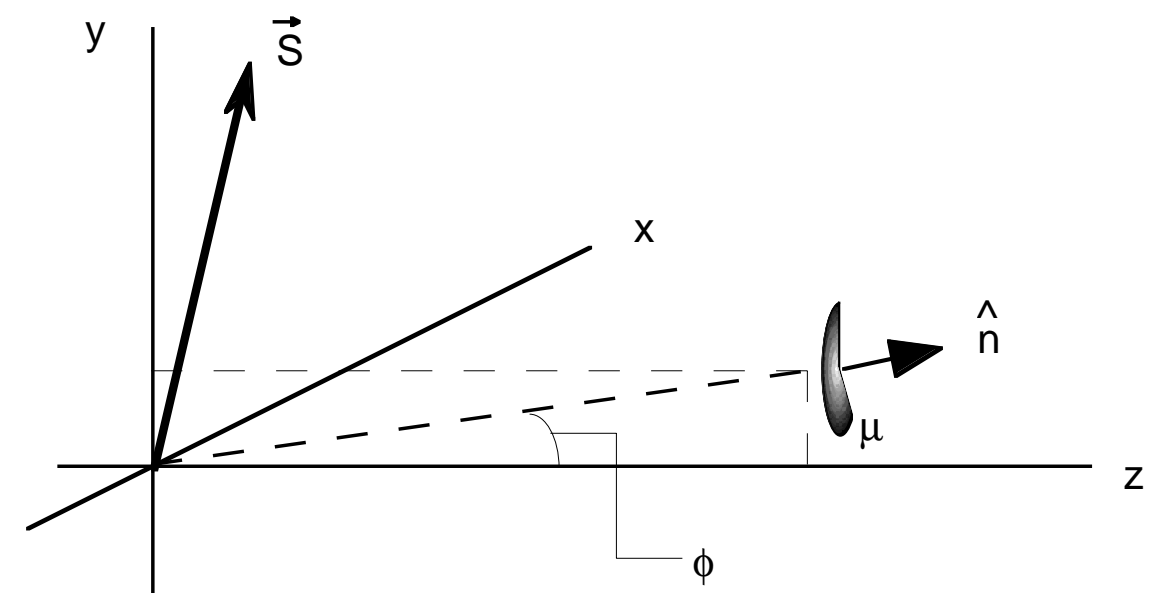

Figure 2: Rotation of spin vector $\vec{S}$ for a "Snake" helical dipole magnet for which $\hat{n}$ is in the vertical plane at an angle $\phi$ with the $z$-axis.

about an axis

$$
\hat{n}=\frac{\hat{x}_{1}+\left(\frac{\kappa}{k}\right) \hat{x}_{3}}{\sqrt{1+\left(\frac{\kappa}{k}\right)^{2}}}
$$

In this case, the unit vector $\hat{n}$ is in the $y-z$ plane, at an angle $\phi=\tan ^{-1}(\kappa / k)$ to the $z$-axis. This case is depicted in Figure 2. The corresponding transformation of the spin vector in physical space is given by the matrix

$$
\begin{aligned}
M_{h s}^{(3)} & =\left(\begin{array}{ccc}
1 & 0 & 0 \\
0 & \cos \phi & \sin \phi \\
0 & -\sin \phi & \cos \phi
\end{array}\right)\left(\begin{array}{ccc}
\cos \mu & -\sin \mu & 0 \\
\sin \mu & \cos \mu & 0 \\
0 & 0 & 1
\end{array}\right)\left(\begin{array}{ccc}
1 & 0 & 0 \\
0 & \cos \phi & -\sin \phi \\
0 & \sin \phi & \cos \phi
\end{array}\right) \\
& =\left(\begin{array}{ccc}
\cos \mu & -\cos \phi \sin \mu & \sin \phi \sin \mu \\
\cos \phi \sin \mu & \cos ^{2} \phi \cos \mu+\sin ^{2} \phi & \cos \phi \sin \phi(1-\cos \mu) \\
-\sin \phi \sin \mu & \cos \phi \sin \phi(1-\cos \mu) & \sin ^{2} \phi \cos \mu+\cos ^{2} \phi
\end{array}\right)
\end{aligned}
$$

where, once again,

$$
\mu=-2 \pi \sqrt{1+\left(\frac{\kappa}{k}\right)^{2}}
$$

It should be pointed out that $M_{h s}^{(3)}$ and $M_{h r}^{(3)}$ were obtained by neglecting the nonlinear terms and the longitudinal component of the helical dipole field. However, they should still prove useful for tracking studies through strong spin resonances in RHIC and for studying operational scenarios. 


$$
\begin{aligned}
& \text { Snake helical dipoles: } \\
& \mathrm{E}:=\text { 24.4.GeV } \quad \gamma:=\frac{\mathrm{E}}{0.938 \mathrm{GeV}} \quad \mathrm{G}:=1.7928 \quad \mathrm{p}:=\sqrt{\mathrm{E}^{2}-(0.938 \cdot \mathrm{GeV})^{2}} \\
& \mathrm{~L}:=2.4 \cdot \mathrm{m} \quad \mathrm{k}:=\frac{2 \cdot \pi}{\mathrm{L}} \quad \mathrm{B} \rho:=\frac{10}{2.9979} \cdot \frac{\mathrm{p}}{\mathrm{GeV}} \cdot \text { tesla } \cdot \mathrm{m} \\
& \kappa(B):=(1+G \cdot \gamma) \cdot \frac{B}{B \rho} \quad \mu_{0}(B):=-2 \cdot \pi \cdot \sqrt{1+\left(\frac{\kappa(B)}{k}\right)^{2}} \quad \phi_{0}(B):=\operatorname{atan}\left(\frac{\kappa(B)}{k}\right) \\
& \mathrm{M}_{\mathrm{hs}}(\mu, \phi):=\left[\begin{array}{ccc}
\cos (\mu) & -\cos (\phi) \cdot \sin (\mu) & \sin (\phi) \cdot \sin (\mu) \\
\cos (\phi) \cdot \sin (\mu) & \cos (\phi)^{2} \cdot \cos (\mu)+\sin (\phi)^{2} & \sin (\phi) \cdot \cos (\phi) \cdot(1-\cos (\mu)) \\
-\sin (\phi) \cdot \sin (\mu) & \sin (\phi) \cdot \cos (\phi) \cdot(1-\cos (\mu)) & \sin (\phi)^{2} \cdot \cos (\mu)+\cos (\phi)^{2}
\end{array}\right] \\
& \text { Complete Snake: } \\
& M_{s}\left(B_{1}, B_{2}\right):=M_{h s}\left(\mu_{0}\left(-B_{1}\right), \phi_{0}\left(-B_{1}\right)\right) \cdot M_{h s}\left(\mu_{0}\left(-B_{2}\right), \phi_{0}\left(-B_{2}\right)\right) \cdot M_{h s}\left(\mu_{0}\left(B_{2}\right), \phi_{0}\left(B_{2}\right)\right) \cdot M_{h s}\left(\mu_{0}\left(B_{1}\right), \phi_{0}\left(B_{1}\right)\right) \\
& \text { Solve for fields: } \quad \text { B }_{1}:=-1.25 \text {.tesla } \quad B_{2}:=4 \text {.tesla } \\
& \text { Given } \\
& \mathrm{M}_{\mathrm{s}}\left(\mathrm{B}_{1}, \mathrm{~B}_{2}\right)_{0,0}=0 \quad \mathrm{M}_{\mathrm{s}}\left(\mathrm{B}_{1}, \mathrm{~B}_{2}\right)_{0,1}=0 \quad \mathrm{M}_{\mathrm{s}}\left(\mathrm{B}_{1}, \mathrm{~B}_{2}\right)_{0,2}=1 \\
& \mathrm{M}_{\mathrm{s}}\left(\mathrm{B}_{1}, \mathrm{~B}_{2}\right)_{1,0}=0 \quad \mathrm{M}_{\mathrm{s}}\left(\mathrm{B}_{1}, \mathrm{~B}_{2}\right)_{1,1}=-1 \quad \mathrm{M}_{\mathrm{s}}\left(\mathrm{B}_{1}, \mathrm{~B}_{2}\right)_{1,2}=0 \\
& \mathrm{M}_{\mathrm{s}}\left(\mathrm{B}_{1}, \mathrm{~B}_{2}\right)_{2,0}=1 \quad \mathrm{M}_{\mathrm{s}}\left(\mathrm{B}_{1}, \mathrm{~B}_{2}\right)_{2,1}=0 \quad \mathrm{M}_{\mathrm{s}}\left(\mathrm{B}_{1}, \mathrm{~B}_{2}\right)_{2,2}=0 \\
& \left(\begin{array}{l}
\mathrm{Bb} 1 \\
\mathrm{Bb} 2
\end{array}\right):=\text { Find }\left(\mathrm{B}_{1}, \mathrm{~B}_{2}\right) \quad \mathrm{Bb} 1=-1.231 \cdot \text { tesla } \quad \mathrm{Bb} 2=3.954^{\cdot} \text { tesla } \\
& \mathrm{M}_{\mathrm{S}}(\mathrm{Bb} 1, \mathrm{Bb} 2)=\left(\begin{array}{ccc}
0 & 0 & 1 \\
0 & -1 & 0 \\
1 & 0 & 0
\end{array}\right)
\end{aligned}
$$

Figure 3: Mathcad calculation of complete Snake using matrices described in the text.

A complete RHIC Snake is made up of four right-handed $(k>0)$ helical dipole magnets where $\kappa_{4}=-\kappa_{1}$ and $\kappa_{3}=-\kappa_{2}$, and where $\kappa_{1}$ and $\kappa_{2}$ are of opposite sign. One would like the overall spin rotation matrix of the complete Snake to be that of a $180^{\circ}$ rotation about a $45^{\circ}$ axis in the horizontal plane. The $3 \times 3$ matrix for the rotation of the spin vector would just be

$$
\left(\begin{array}{ccc}
0 & 0 & 1 \\
0 & -1 & 0 \\
1 & 0 & 0
\end{array}\right)
$$

Figure 3 shows the result of a Mathcad calculation using two independent fields $B_{4}=-B_{1}=1.23 \mathrm{~T}$, and $B_{2}=-B_{3}=3.95 \mathrm{~T}$, where a negative field means the field direction is "down" at the entrance/exit of the magnet. Using Luccio's program 


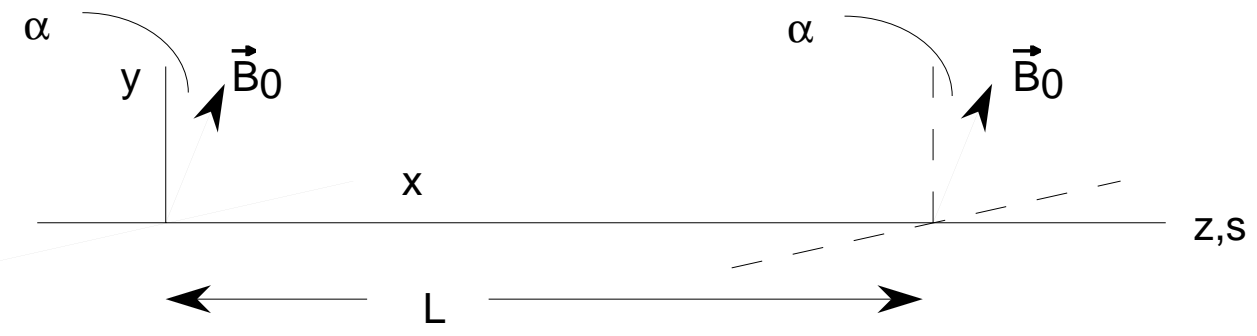

Figure 4: Complete $\left(360^{\circ}\right)$ helical dipole with arbitrary entrance and exit angle $\alpha$.

$\mathrm{SNIG}^{6}$, which includes the full nonlinear helical field of the magnet, as well as modeled end effects, the required values of $B_{1}$ and $B_{2}$ for a Snake were found to be $1.191 \mathrm{~T}$ and $3.864 \mathrm{~T}$, approximately $2-3 \%$ agreement with the above.

To summarize, the spin vector $\vec{S}=\left(S_{x}, S_{y}, S_{z}\right)$ transforms through a helical dipole magnet of length $L$ and central field $B_{0}$ according to the matrix:

$$
\begin{aligned}
& \left(\begin{array}{ccc}
\cos \alpha & \sin \alpha & 0 \\
-\sin \alpha & \cos \alpha & 0 \\
0 & 0 & 1
\end{array}\right) \times \\
& \left(\begin{array}{ccc}
\cos \mu & -\cos \phi \sin \mu & \sin \phi \sin \mu \\
\cos \phi \sin \mu & \cos ^{2} \phi \cos \mu+\sin ^{2} \phi & \cos \phi \sin \phi(1-\cos \mu) \\
-\sin \phi \sin \mu & \cos \phi \sin \phi(1-\cos \mu) & \sin ^{2} \phi \cos \mu+\cos ^{2} \phi
\end{array}\right)\left(\begin{array}{ccc}
\cos \alpha & -\sin \alpha & 0 \\
\sin \alpha & \cos \alpha & 0 \\
0 & 0 & 1
\end{array}\right)
\end{aligned}
$$

where $\alpha$ is the angle the field makes from the vertical ( $y$-axis) toward the horizontal ( $x$-axis) (see Figure 4$)$ at the entrance/exit of the magnet, and

$$
\begin{aligned}
\mu & =-2 \pi \sqrt{1+\left(\frac{\kappa}{k}\right)^{2}} \\
\phi & =\tan ^{-1}(\kappa / k) \\
\kappa & =(1+G \gamma) \frac{B_{0}}{B \rho} \\
k & \left.=\frac{2 \pi}{L} \text { (right }- \text { handed }\right) \\
& \left.=-\frac{2 \pi}{L} \text { (left }- \text { handed }\right)
\end{aligned}
$$

For a Snake magnet, $\alpha=0$. For a Rotator magnet, $\alpha=\pi / 2$. If coded into SPINK, for example, then rotational misalignment errors can be studied by using different values of $\alpha$, while powering errors and operational adjustments can be studied by varying $B_{0}$ for each magnet.

\footnotetext{
${ }^{6}$ A. Luccio, SN/8.
} 